\title{
Response
}

\section{Metformin Preserves Peripheral Nerve Damage with Comparable Effects to Alpha Lipoic Acid in Streptozotocin/High-Fat Diet Induced Diabetic Rats (Diabetes Metab J 2020;44:842-53)}

\author{
Sun Hee Kim, Tae Sun Park, Heung Yong Jin \\ Division of Endocrinology and Metabolism, Department of Internal Medicine, Jeonbuk National University Medical School and Research Institute of Clinical \\ Medicine of Jeonbuk National University-Biomedical Research Institute of Jeonbuk National University Hospital, Jeonju, Korea
}

We appreciate Professor Bo-Kyung Koo for her interest and valuable comments regarding our recently published article, "Metformin preserves peripheral nerve damage with comparable effects to alpha lipoic acid in streptozotocin/high-fat diet induced diabetic rats," which was published in Diabetes \& Metabolism Journal [1].

First, we agree with Professor Koo's opinion that there was no measurement of pro-inflammatory and inflammatory markers to explain the exact mechanism of the neuroprotective effect of metformin in this study. This study was performed to determine whether metformin is harmful in progression of diabetic peripheral neuropathy (DPN) in relation to vitamin B 12 deficiency. Therefore, we did not focus on the mechanism of the neuroprotective effect in this study. We assessed important mediators and oxidative stress in DPN such as tumor necrosis factor $\alpha$ and glutathione levels with vitamin B 12; however, pro-inflammatory/inflammatory markers are needed to support the beneficial role of metformin in DPN management, as Professor Koo commented. Therefore, measurement of diverse oxidative stress markers in target tissue and well known pro-inflammatory and inflammatory markers including C-reactive protein, interleukin series, and nuclear factor- $\kappa \mathrm{B}$ pathways would provide greater support for the therapeutic mechanisms of metformin.
Second, Professor Koo suggested that inclusion of another anti-diabetic medication arm in the study would have allowed separation of the effect of glucose control by metformin from the benefit of neuroprotection of metformin independent of its glucose-lowering effect. We also agree with this suggestion; however, this concept is out of the scope of this study, as we explained above, a limitation of this article. To exclude the glucose-lowering benefit from metformin treatment in neuroprotection, a second group with similar glucose control must be analyzed. In addition, the adequacy of glucose-lowering agents for neuropathy experiments should be determined. As Professor Koo commented, metformin was reported to be protective against paclitaxel-induced neuropathic pain in a non-diabetic rat model [2], which supports the neuroprotective benefit of metformin without a glucose-lowering effect. Therefore, if there had been another neuropathy group such as chemotherapeutic drug or toxin-induced neuropathy group, our results would be more meaningful. In this study, we suggest that metformin provides beneficial roles in preservation of peripheral nerves in diabetic rats, to a comparable degree to alpha lipoic acid. There have been inconsistent effects on DPN from metformin treatment regarding vitamin B12 deficiency in management of diabetic patients [3]. Therefore, we suggest that the positive effects of metformin on peripheral nerves need to be
Corresponding author: Heung Yong Jin (DD https://orcid.org/0000-0002-1841-2092 Division of Endocrinology and Metabolism, Department of Internal Medicine, Jeonbuk National University Medical School, 20 Geonji-ro, Deokjin-gu, Jeonju 54907, Korea E-mail: mdjinhy@jbnu.ac.kr
This is an Open Access article distributed under the terms of the Creative Commons Attribution Non-Commercial License (https://creativecommons.org/licenses/by-nc/4.0/) which permits unrestricted non-commercial use, distribution, and reproduction in any medium, provided the original work is properly cited. 
considered in management of patients with DPN.

\section{CONFLICTS OF INTEREST}

No potential conflict of interest relevant to this article was reported.

\section{REFERENCES}

1. Kim SH, Park TS, Jin HY. Metformin preserves peripheral nerve damage with comparable effects to alpha lipoic acid in streptozotocin/high-fat diet induced diabetic rats. Diabetes Metab J 2020;44:842-53.

2. Hacimuftuoglu A, Mohammadzadeh M, Taghizadehghalehjoughi A, Taspinar N, Togar B, Nalci KA, et al. The analgesic effect of metformin on paclitaxel-induced neuropathic pain model in rats: by considering pathological results. J Cancer Res Ther 2020;16:34-9.

3. Aroda VR, Edelstein SL, Goldberg RB, Knowler WC, Marcovina SM, Orchard TJ, et al. Long-term metformin use and vitamin b12 deficiency in the diabetes prevention program outcomes study. J Clin Endocrinol Metab 2016;101:1754-61. 